\title{
Optical chemsensors based on etched fibre Bragg gratings in D-shape and multimode fibres
}

\author{
Kaiming Zhou, Xianfeng Chen, Lin Zhang, Ian Bennion \\ Photonics Research Group, Electronic Engineering, Aston University, Birmingham, B4 7ET, UK
}

\begin{abstract}
We present an implementation of high-sensitivity optical chemsensors based on FBGs UV-inscribed in D-shape and multimode fibres and sensitized by HF-etching treatment, demonstrating a capability of detecting chemical concentration changes as small as $<0.5 \%$.
\end{abstract}

Keywords: fibre Bragg grating, tilted fibre grating, chemsensor, chemical etching, multimode fibre, D-shape fibre

\section{INTRODUCTION}

Over the last decade, fibre Bragg gratings (FBGs) have been utilized as optical sensors to measure a wide range of physical parameters including temperature, strain, pressure, loading, bending, etc. [1-3]. Since the light coupling takes place between well-bound core modes that are screened from the influence of the surrounding-medium refractive index (SRI) by the cladding, normal FBGs are intrinsically insensitive to SRI. In contrast, long-period grating (LPG) couples the light between the core and cladding modes, the cladding acts as an interface with the external medium making LPG intrinsically sensitive to SRI. To date, a number of SRI sensors have been realised using LPG structures to measure concentrations of some chemicals [4]. Despite high SRI sensitivity, LPGs exhibit several disadvantages as deployable devices, such as higher temperature and bending cross-sensitivities, broad resonance and measurement in transmission only. In this paper, we report an implementation of optical chemsensor devices based on FBG structures in D-shape fibre (DF) and tilted FBGs (TFBGs) in multimode fibre (MMF), sensitised by hydrofluoric acid (HF) etching. The devices have been used to measure the concentrations of sugar solutions, demonstrating sensitivities as high as $0.02 \mathrm{~nm} / \%$. These FBG based chemsensors exhibit device advantages over equivalent LPFG structures.

\section{FBG CHEMSENSORS IN D-SHAPE FIBRE}

The DF used in our investigation was commercially purchased from KVH in the US. Fig. 1a shows the cross-sectional profile of the DF. The distance between the flat surface of the cladding and the core is $\sim 13.5 \mu \mathrm{m}$. The core is elliptical with a short axis of $2.5 \mu \mathrm{m}$ and a long axis of $4.5 \mu \mathrm{m}$, and is surrounded by a fluorine-doped thin elliptical inner cladding layer. In order to effectively control the etched cladding profile of the FBGs, we first evaluated the etching rate using HF at $10 \%$ concentration. Twenty DF samples were immersed in the HF bath and were withdrawn in turn every 10min. The samples with differently etched claddings were then examined and measured using a microscope. Fig. $1 \mathrm{~b}$ plots the etched cladding thickness against etching time, showing a rate of $\sim 0.068 \mu \mathrm{m} / \mathrm{min}$. The cladding on the flat side was almost completely removed after $\sim 175$ minutes, leaving the core exposing to air on this side.

The spectral evolution of the DF FBGs during HF etching was then investigated. The FBGs were UV-inscribed in the hydrogenated D-fibre employing a frequency doubled Ar laser and a two-beam holographic fabrication system. When the DF FBG was submerged to HF bath, the spectrum was monitored in-situ using an EDFA source and an optical spectrum analyser. We noted that the spectrum of the FBG remained intact for the etching period from 10 to $140 \mathrm{~min}$, since the core mode was still well bounded by the cladding. The Bragg response started to change when the thickness of the residual cladding layer on the flat side of the DF was reduced to just a few microns, thereby allowing the evanescent field penetrating the surrounding medium. With continuous etching, the Bragg resonance shifted towards the shorter wavelengths dramatically, entering the SRI sensitive regime. Fig. 1c shows the spectral evolution of a DF FBG in the beginning of this sensitising regime period from 140 to $168 \mathrm{~min}$.

Two DF FBGs (G1 and G2) HF-etched for 155min and 170min respectively were selected for the implementation of optical chemsensors to measure the concentrations of sugar solutions. The sugar solutions with concentrations ranging from 0\% to 60\% were prepared in-house. The etched DF Bragg gratings were immersed in turn into each sugar solution and their Bragg wavelengths measured using the broadband light source and optical spectrum analyser. Since the sensing mechanism of the etched DF grating is based on the grating response to the change of SRI, we first calibrated the 
measurement using the relationship between sugar concentration and refractive index provided by using the data in reference [5], and the calibration is plotted in Fig. 2a.

(a)

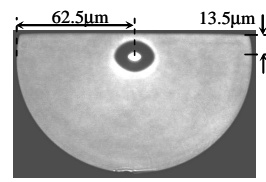

(b)

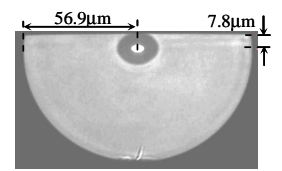

(c)

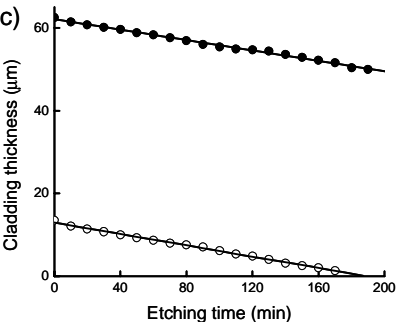

(a)

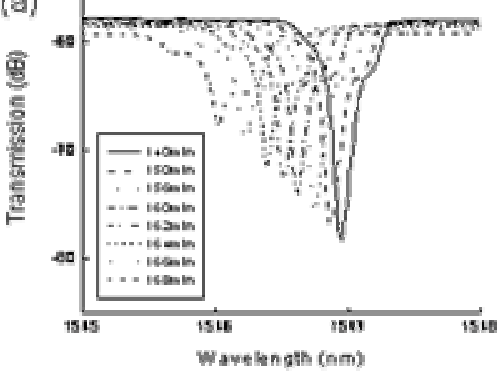

Fig. 1. (a) The cross-section images of the D-fibres (pristine and HF etched for $\sim 80$ minutes); (b) cladding thickness against etching time; (c) spectral evolution of the D-fibre FBG under etching from 140 to $168 \mathrm{~min}$.

For sugar concentration changes from $0 \%$ to $60 \%$, the refractive index increases more or less linearly from 1.33 to 1.44 . Fig. 2b displays the SRI sensing characteristics of three DF FBGs: G1 and G2, and one un-etched FBG. It is clear from this figure that the un-etched grating is totally insensitive to SRI whereas the Bragg wavelengths of G1 and G2 red-shift at different rates with increasing SRI. The deeper etched grating G2 exhibits a much higher SRI sensitivity than the less etched G1. If we define the SRI sensitivity as the wavelength shift induced by $1 \%$ SRI change, the maximum sensitivities exhibited by G1 and G2 are $0.03 \mathrm{~nm} / \%$ and $0.11 \mathrm{~nm} / \%$, respectively. The latter is almost four times that of the former. Using Fig. 2a, we can convert the SRI sensitivity to the sugar concentration sensitivity. Defining this as the Bragg resonance shift induced by $1 \%$ sugar concentration change, we have sensitivities of $0.005 \mathrm{~nm} / \%$ and $0.02 \mathrm{~nm} / \%$ for G1 and G2, respectively. Thus, for practical applications, up to 5\% concentration change in sugar concentration can be easily detected by G2 using a standard optical interrogation system with an optical resolution of $\sim 0.1 \mathrm{~nm}$ and $0.5 \%$ change with a resolution of $0.01 \mathrm{~nm}$.
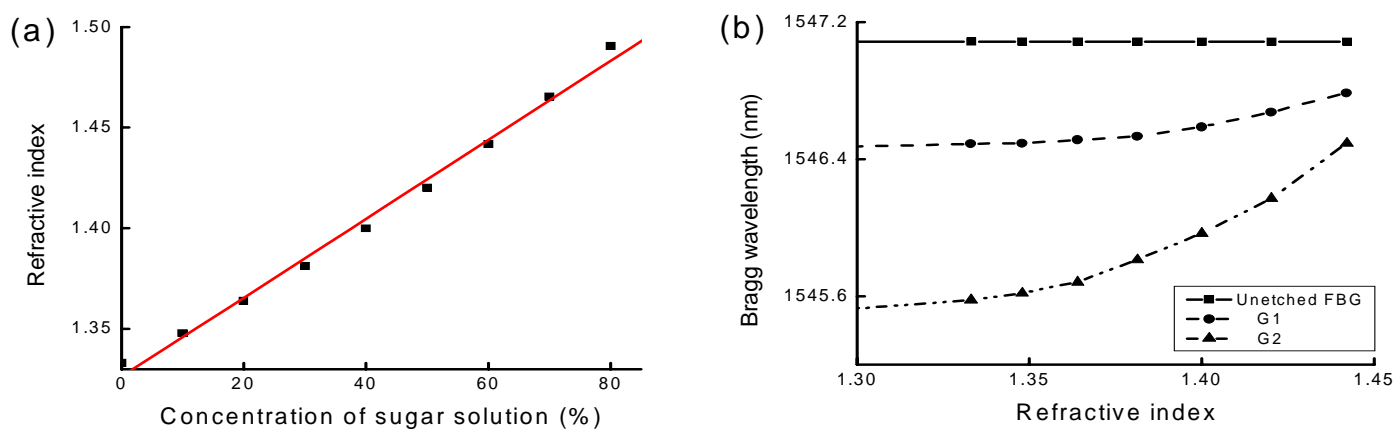

Fig. 2 (a) Calibration plot of refractive index against concentration of sugar solution; (b) Sensitivity plots of an unetched DF FBG and etched G1 and G2.

\section{TFBG chemsensors in multimode fibre}

The MMF used in our experiment is the standard graded index fibre with a N.A. of 0.22 and a core diameter of $50 \mu \mathrm{m}$. The FBG structures were UV-inscribed in the $\mathrm{H}_{2}$-loaded MMF pieces by the scanning phase mask technique in 
conjunction with a frequency doubled Ar laser. In order to investigate the light couplings between the symmetrical and asymmetrical core and cladding modes, both normal and tilted structures were fabricated in MMF. To realize the latter structure, the phase mask was tilted with respect to the fibre axis in the UV-inscription system. In confirmation of the tilted structures, we examined the MMF gratings under the microscope with high magnification.

Fig. 3a, b and c depict typical transmission spectra of FBGs UV-inscribed when the phase mask was tilted at $0^{\circ}, 0.7^{\circ}$ and $3.5^{\circ}$, showing not only distinctive characteristics but also a clear transition of the light coupling from core-core to core-cladding modes. For the normal structure, the core mode field overlap integral is much larger for symmetric $(\mathrm{m}=0$, $2, \ldots)$ modes than for asymmetric $(\mathrm{m}=1,3, \ldots)$ ones. In our characterisation, since the light was launched to the MMF using a broadband source with an SMF pigtail, we can regard that the light was traveling mainly in the fundamental mode. Thus, the resonances in Fig. 3a are corresponding to the core-core mode coupling of $0 \rightarrow 0,2, \ldots, 10$. The lowest order mode, $L P_{c o-c o}^{0 \rightarrow 0}$, is the most strongly coupled, resulting in $\sim 23 \mathrm{~dB}$ Bragg reflection at $1579.9 \mathrm{~nm}$, whereas the coupling to the high modes $(n=6,8$ and 10$)$ are so weak to appear. When the grating is slightly tilted $\left(0.7^{\circ}\right)$, the mode coupling is still confined in the core modes, however, the tilted structure enhances the coupling to asymmetric modes. Thus, we see the extra resonances in Fig. $3 \mathrm{~b}$, resulted from the mode coupling: $0 \rightarrow 1,3, \ldots, 9$. The lowest mode is still the strongest, but the strength of the resonance attends decreasing linearly with increasing mode order. At $3.5^{\circ}$, one can see a clear transition of the mode coupling from core-core to core-cladding, as shown in Fig. 3c. The resonances on the right-hand side of Fig.3c still correspond to the core-core mode coupling, whereas the resonances on the left-hand side are resulted from core-cladding coupling. This was verified by immersing the grating in the index matching gel; the cladding resonances were disappeared and replaced by a smooth envelope profile representing the coupling to a continuum of radiation modes.
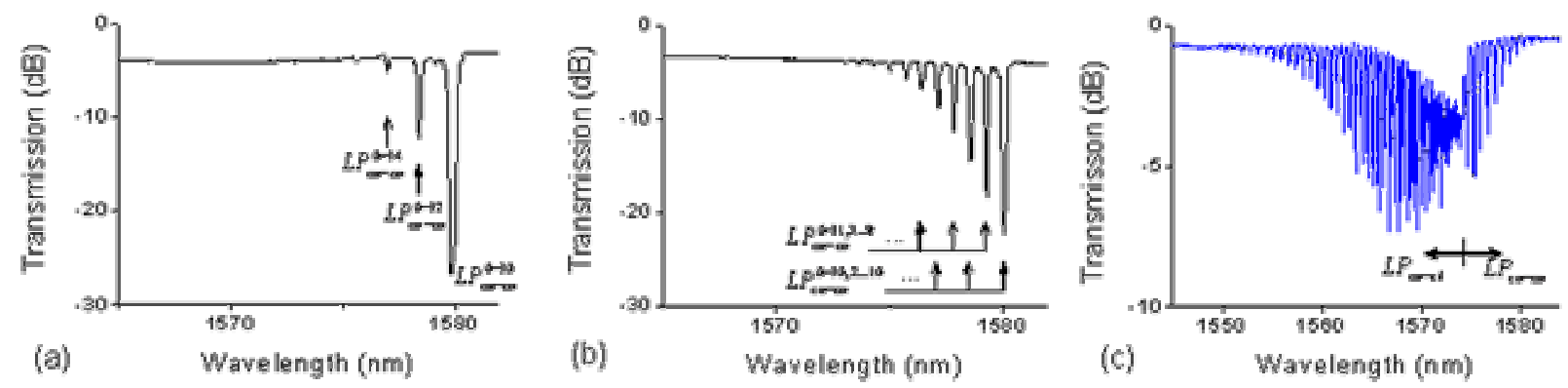

Fig. 3 Transmission spectra of (a) non-tilted, (b) 0.7 -tilted and (c) 3.5 -tilted FBGs in MMF.

Since the core modes in MMF are also well-bound and screened from the SRI influence by the cladding, it is necessary to sensitise MMF FBGs by HF-etching. Several grating samples were etched using an HF acid of $15 \%$ concentration and their spectra were also monitored in-situ using the EDFA and spectrum analyser. We found the Bragg resonances of MMF FBGs were remained intact for the first 160min of the etching process and, after this point, the resonances blue-shifted with acceleration. Fig. 4a shows the spectral evolution of the $0.7^{\circ}$-tilted grating for the etching period from $160 \mathrm{~min}$ to $252 \mathrm{~min}$. We noticed that after the cladding being etched off after $\sim 165 \mathrm{~min}$, the high order modes started blue-shifting whereas the low order modes were still static. From 160 to $220 \mathrm{~min}$, the three lowest order modes resonances were almost unchanged and they started moving after 220min at increasing rates with increasing etching time and mode order. It was estimated that the core size was reduced to $\sim 12 \mu \mathrm{m}$ after $252 \mathrm{~min}$, confining only two/three modes.

The etched $0.7^{\circ}$-tilted FBG with $12 \mu \mathrm{m}$ remaining diameter was investigated for SRI sensitivity also by measuring the sugar solutions with concentrations ranging from $0 \%$ to $60 \%$. Fig. $4 \mathrm{~b}$ plots the SRI-induced wavelength shifts of the two lowest order modes $\left(L P_{c o-c o}^{0 \rightarrow 0}, L P_{c o-c o}^{0 \rightarrow 1}\right)$. It is clear from the figure that the higher order core mode is more sensitive to 
SRI change than the lower order one; the former shifted $5.35 \mathrm{~nm}$ whereas the latter only $2.56 \mathrm{~nm}$ over the index range 1.333-1.442. The maximum sensitivities exhibited by $L P_{c o-c o}^{0 \rightarrow 0}$ and $L P_{c o-c o}^{0 \rightarrow 1}$ are $0.70 \mathrm{~nm} / \%$ and $1.51 \mathrm{~nm} / \%$, respectively. The latter is more than 2 times that of the former. Using the calibrating data of refractive index to concentration, we estimate that the higher order resonance shift induced by $1 \%$ sugar concentration change is $\sim 0.23 \mathrm{~nm} / \%$ in $1.420-1.442$ range. Thus, the sugar concentration change as small as $0.43 \%$ can readily be detected by this grating using a standard interrogation system with an optical resolution of $0.1 \mathrm{~nm}$, and $\sim 0.04 \%$ change may be possible to detect by using a tunable laser with $0.01 \mathrm{~nm}$ resolution if the noise effect can be eliminated effectively.

(a)

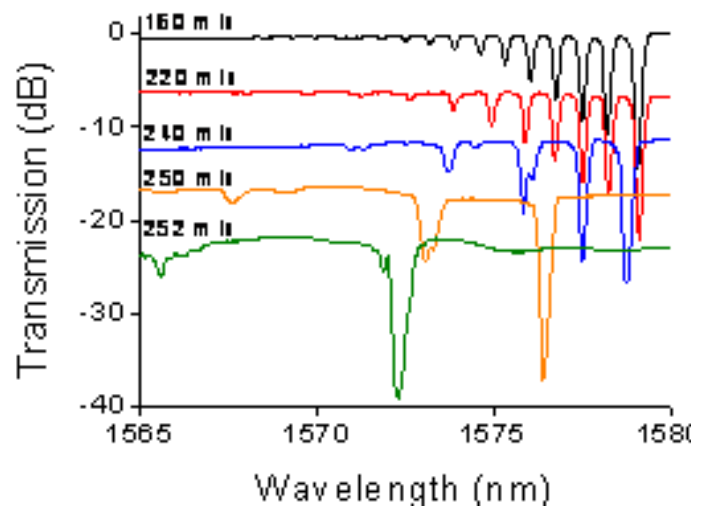

(b)

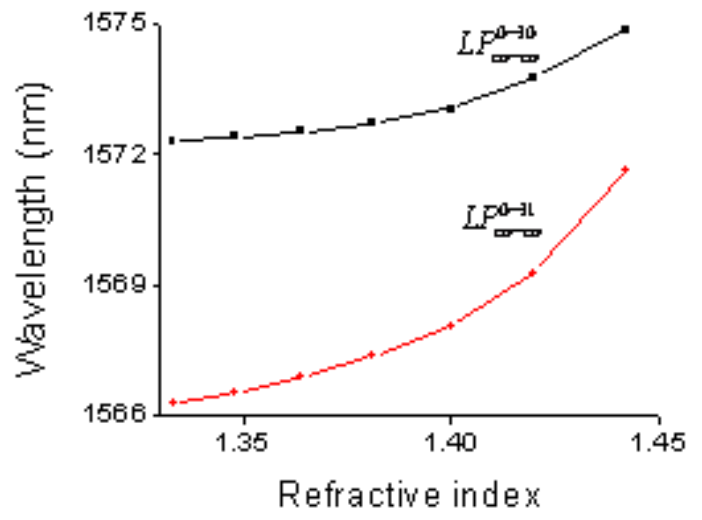

Fig. 4(a) The spectral evolution of $0.7^{\circ}$-tilted FBG in MMF during HF-etching process from 160 to $252 \mathrm{~min}$; (b) wavelength shifts of the two lowest order modes $\left(L P_{c o-c o}^{0 \rightarrow 0}, L P_{c o-c o}^{0 \rightarrow 1}\right)$ of the etched TFBG against SRI change.

\section{Conclusions}

We have demonstrated two configurations of optical chemsensors based on FBGs in DF and MMF whose SRI sensitivities have been significantly improved by HF-etching process. The etched devices were used to measure concentrations of sugar solution, showing maximum detection sensitivities of $0.11 \mathrm{~nm} / \%$ and $0.23 \mathrm{~nm} / \%$, respectively. As potential devices, chemical concentration changes as small as $0.05 \%$ may be detected for biochemical and environmental sensing applications.

\section{REFERENCE}

1. A. Othonos and K. Kalli, Fiber Bragg Gratings: Fundamentals and Applications in Telecommunications and Sensing, Artech House, London, 1999.

2. L. Zhang, W. Zhang, and I. Bennion, "In-fiber grating optic sensors", in Fiber Optic Sensors, ed. by F. T. S. Yu \& S. Yin, , pp. 123-181, Marcel Dekker, New York, 2002.

3. M.A.Davis and a.D.kersey, "Fiber Bragg grating sensors for infrastructure sensing”. OFC'97 pp 177-178, Dallas, TX, USA, 1997,.

4. X.Shu, L.Zhang and I.Bennion, "Sensitivity characteristics of long-period fiber gratings", IEEE J. Lightwave Technol. 2002, 20, pp. 255-256

5. R.C.West (Ed.) "Handbook of chemistry and physics" (CRC, Boca Raton, FL, 1981, 61 ${ }^{\text {st }}$ edn) 